\title{
Article
}

\section{Development of a Fundus Image-Based Deep Learning Diagnostic Tool for Various Retinal Diseases}

\author{
Kyoung Min Kim ${ }^{1,+}$, Tae-Young Heo ${ }^{2,+}{ }^{\mathbb{D}}$, Aesul Kim ${ }^{3}$, Joohee Kim ${ }^{3}$, Kyu Jin Han ${ }^{4}$, Jaesuk Yun ${ }^{3, *}$ \\ and Jung Kee Min ${ }^{4, *(D)}$
}

1 Data Scientist Team, BEGAS Inc., Sejong-daero 39, Jung-gu, Seoul 04513, Korea; ganaaml2002@naver.com

2 Department of Information and Statistics, Chungbuk National University, Chungdae-ro 1, Seowon-gu, Cheongju-si, Chungbuk 28644, Korea; theo@cbnu.ac.kr

3 Laboratory of Pharmacology, College of Pharmacy and Medical Research Center, Chungbuk National University, 194-31 Osongsaengmyeong 1-ro, Osong-eup, Heungdeok-gu, Cheongju-si, Chungbuk 28160, Korea; aeseulkim@daum.net (A.K.); joohyee4u@naver.com (J.K.)

4 Department of Ophthalmology, Ulsan University Hospital, College of Medicine, University of Ulsan, 877, Bangeojinsunhwando-ro, Dong-gu, Ulsan 44033, Korea; hankj229@hanmail.net

* Correspondence: jyun@chungbuk.ac.kr (J.Y.); jkmin@uuh.ulsan.kr (J.K.M.); Tel.: +82-43-261-2827 (J.Y.); +82-52-250-7170 (J.K.M.); Fax: +82-43-268-2732 (J.Y.)

+ These authors equally contributed to this study.

check for updates

Citation: Kim, K.M.; Heo, T.-Y.; Kim, A.; Kim, J.; Han, K.J.; Yun, J.; Min, J.K. Development of a Fundus Image-Based Deep Learning Diagnostic Tool for Various Retinal Diseases. J. Pers. Med. 2021, 11, 321. https://doi.org/10.3390/jpm11050321

Academic Editor: Jorge Luis Espinoza

Received: 7 March 2021

Accepted: 18 April 2021

Published: 21 April 2021

Publisher's Note: MDPI stays neutral with regard to jurisdictional claims in published maps and institutional affiliations.

Copyright: (c) 2021 by the authors. Licensee MDPI, Basel, Switzerland. This article is an open access article distributed under the terms and conditions of the Creative Commons Attribution (CC BY) license (https:/ / creativecommons.org/licenses/by/ $4.0 /)$.

\begin{abstract}
Artificial intelligence (AI)-based diagnostic tools have been accepted in ophthalmology. The use of retinal images, such as fundus photographs, is a promising approach for the development of AI-based diagnostic platforms. Retinal pathologies usually occur in a broad spectrum of eye diseases, including neovascular or dry age-related macular degeneration, epiretinal membrane, rhegmatogenous retinal detachment, retinitis pigmentosa, macular hole, retinal vein occlusions, and diabetic retinopathy. Here, we report a fundus image-based AI model for differential diagnosis of retinal diseases. We classified retinal images with three convolutional neural network models: ResNet50, VGG19, and Inception v3. Furthermore, the performance of several dense (fully connected) layers was compared. The prediction accuracy for diagnosis of nine classes of eight retinal diseases and normal control was $87.42 \%$ in the ResNet50 model, which added a dense layer with 128 nodes. Furthermore, our AI tool augments ophthalmologist's performance in the diagnosis of retinal disease. These results suggested that the fundus image-based AI tool is applicable for the medical diagnosis process of retinal diseases.
\end{abstract}

Keywords: artificial intelligence; class activation map; convolutional neural network; fundus photograph; retinal diseases

\section{Introduction}

Since the Food and Drug Administration's approval of the IDx-DR system for diabetic retinopathy diagnosis [1], deep learning-based tools have been extensively developed in ophthalmology. Various retinal diseases have useful features for the development of image-based diagnostic systems. For example, drusen or choroidal neovascularization (CNV), subretinal hemorrhage, and vascular leakage are the pathophysiology of dry or neovascular age-related macular degeneration (dAMD or nAMD). These pathologies represent potential photographic sources for the development of artificial intelligence (AI) diagnostic tools [2]. Moreover, other retinal diseases also have diagnostic markers, allowing ophthalmologists to identify retinal changes and differentiates conditions with peer review [3]. Diabetic retinopathy (DR) is characterized by microaneurysms, capillary nonperfusion, and ischemia within the retina [4,5]. In DR, block of blood vessels and irregular in diameter [6] associated with fluid leakage and hemorrhage, which may induce vision loss. In addition, neovascularization is a pathological process in DR [7]. The epiretinal membrane (ERM) is an epimacular membranous tissue, which develops in 
relation to proliferative disease, inflammation, uveitis, or trauma, and other causes. ERM can lead to tangential traction, causing retinal changes, such as retinal layer thickening, surface wrinkling, and/or nerve fiber layer fibrillation, which cause decreased visual acuity (VA) and metamorphopsia [8-12]. Rhegmatogenous retinal detachment (RRD) is a vision-threatening disease caused by retinal break, which allows vitreous fluid to escape into the subretinal space. As a result, the sensory retina detaches from the retinal pigment epithelium (RPE) [13]. Retinitis pigmentosa (RP) is an inherited retinal disease characterized by progressive loss of photoreceptors and eventual visual loss $[14,15]$. The clinical diagnosis of RP is based on the presence of nyctalopia, visual field constriction, bone spicule pigmentation, and reduction in electroretinogram (ERG) amplitude. Macular hole $(\mathrm{MH})$ is a round full-thickness opening in the foveal center. In most cases, it is idiopathic, that is, due to abnormal vitreofoveal traction. The advent of optical coherence tomography (OCT), which showed the partially detached posterior hyaloid, enhanced the understanding of $\mathrm{MH}$ formation [16,17]. Retinal vein occlusion (RVO) is a heterogeneous group of disorders that commonly have impaired venous return from the retinal circulation. If the occlusion occurs within or posterior to the optic nerve head, it is labeled central RVO (CRVO), and any obstruction within a tributary is a branch RVO (BRVO). Retinal images with CRVO show the features of venous tortuosity, scattered flame-shaped retinal hemorrhages, and/or cotton wool spots in all four quadrants. Patients with BRVO present with a wedge-shaped pattern of intraretinal hemorrhage in the area drained by the occluded vein $[18,19]$.

The characteristic features of such various retinal diseases can be confirmed in fundus photographs. Therefore, we demonstrated the viability of fundus imaging for the application of a deep learning-based AI diagnostic tool and tested the performance of the developed AI tool for differentially diagnosing retinal diseases, showing the abovementioned characteristic fundus features. Furthermore, we compared the diagnostic performance of ophthalmology residents who used or did not use the AI tool.

\section{Materials and Methods}

\subsection{Subjects}

To select the study groups of patients with retinal diseases, the medical records of patients aged $>20$ years who had been diagnosed with eight retinal diseases (dAMD, nAMD, DR, ERM, RRD, RP, MH, and RVO) between 1 January 2015, and 30 June 2020, at the Department of Ophthalmology of Ulsan University Hospital, Ulsan, Republic of Korea, were retrospectively reviewed. All subjects (628 eyes) underwent a complete ophthalmic examination that included the best-corrected VA assessment, noncontact tonometry (CT-1P; Topcon Corporation, Tokyo, Japan), fundus examination using slit-lamp ophthalmoscopy, fundus photography (TRC-NW8, Topcon Corporation, Tokyo, Japan), and swept-source OCT (DRI OCT-1 Atlantis or Triton; Topcon Corporation, Tokyo, Japan), and/or fluorescein angiography (FA) and indocyanine green angiography (ICGA) (Heidelberg Retina Angiograph Spectralis; Heidelberg Engineering, Heidelberg, Germany). nAMD (79 eyes) was diagnosed by fundus examination, including one or more features, such as subretinal and/or intraretinal fluid, subretinal hemorrhage, and retinal pigmented epithelial detachment and intraretinal exudate in the macular area using fundus photography and OCT. $\mathrm{CNV}$ or polypoidal vascular lesions were detected via FA and ICGA. Based on the category of AMD in age-related eye disease [20], drusen types corresponding to categories 2 and 3 were defined as dAMD (58 eyes). DR (95 eyes) was diagnosed based on the medical history and clinical retinal examination. We confirmed several morphological features of $\mathrm{DR}$, including microaneurysms, intraretinal microvascular abnormalities, and neovascularization, by fundus photography and FA. In ERM (99 eyes), the retinal changes were shown as irregular wrinkling, retinal layer thickening and dragging, and ectopic fovea by fundus photography and OCT. RRD (80 eyes) was diagnosed by fundus examination, showing separation between the neurosensory retina and RPE, with an accumulation of liquefied vitreous in the subretinal space through the retinal breaks. We selected only 
fundus photographs to confirm these features in this study. RP (50 eyes) was diagnosed based on the presence of nyctalopia, visual field constriction, specific fundus appearance (bone spicule pigmentation, attenuated retinal vessels, mottling and granularity of RPE, and optic nerve head pallor), and reduction in ERG amplitude. We selected only fundus photographs to confirm specific fundus appearance in this study. MH (49 eyes) was diagnosed by fundus photography and OCT, showing full-thickness retinal opening in the foveal center of various sizes with or without posterior hyaloid (stage 3 and 4). RVO (39 eyes) was diagnosed based on the features of venous tortuosity, scattered or wedge flame-shaped retinal hemorrhages, and/or cotton wool spots using fundus photography. In selecting the normal controls (79 eyes), the medical records of patients who visited the ophthalmology outpatient clinic were also reviewed.

The subjects' demographic distribution of each class is shown in Table 1. When analyzing the difference between groups according to age using the one-way analysis of variance test, $\mathrm{DR}, \mathrm{RRD}, \mathrm{RP}$, and Control are one homogenous subset, and the remaining groups (dAMD, nAMD, ERM, MH, and RVO) consisted of the other homogenous subsets. In the control group, the average age was 56.7, which is close to the average age of all subjects, 61.2 years. In addition, when the gender distribution between each class was confirmed by Pearson's chi-square test, there was no significant difference in gender distribution between each group $(p=0.775)$.

Table 1. Eyes $(n=549)$ were diagnosed with dAMD, nAMD, DR, ERM, RRD, RP, MH, or RVO, and fundus images were collected. Furthermore, 79 eyes from healthy subjects were collected as controls.

\begin{tabular}{|c|c|c|c|c|c|c|c|c|c|}
\hline Disease & dAMD & nAMD & DR & ERM & RRD & $\mathbf{R P}$ & MH & RVO & Control \\
\hline Fundus images $(n)$ & 58 & 79 & 95 & 99 & 80 & 50 & 49 & 39 & 79 \\
\hline \multirow{3}{*}{$\begin{array}{ll}\text { Gender } & \text { Male } \\
& \text { Femal } \\
\text { Age (years) } & \end{array}$} & 27 & 41 & 53 & 53 & 47 & 26 & 31 & 19 & 40 \\
\hline & 31 & 38 & 42 & 46 & 33 & 24 & 18 & 20 & 39 \\
\hline & $69.6 \pm 8.0$ & $69.1 \pm 8.3$ & $53.2 \pm 10.4$ & $63.6 \pm 7.6$ & $54.4 \pm 14.6$ & $53.4 \pm 11.0$ & $64.2 \pm 8.9$ & $67.5 \pm 8.0$ & $56.7 \pm 7.3$ \\
\hline
\end{tabular}

Age (years) are presented as mean \pm standard deviation. dAMD, dry age-related macular degeneration; nAMD, neovascular age-related macular degeneration; DR, diabetic retinopathy; ERM, epiretinal membrane; RRD, rhegmatogenous retinal detachment; RP, retinitis pigmentosa; $\mathrm{MH}$, macular hole; $\mathrm{RVO}$, retinal vein occlusion.

\subsection{Fundus Imaging}

Fundus photography (TRC-NW8 and DRI Triton) provides high-quality 16.2-megapixel images, with a $45^{\circ}$ field of central macular view. All retinal images were reviewed by a retinal specialist (JKM) to ensure that the photographs had sufficiently high quality to adequately visualize the retina. Those with only one disease on one fundus photograph was selected.

\subsection{Augmentation of Data}

To prevent overfitting of AI models and increase the diversity of the dataset, we applied Keras ImageDataGenerator as the data augmentation method. During training, each image was augmented through several methods. Table 2 shows the values of each arguments used in Keras ImageDataGenerator. The value of argument means the range from 0 to the value. (1) Images were cropped randomly on width for approximately $40 \%$ of their size, (2) images were cropped randomly on height for approximately $20 \%$ of their size, (3) images were zoomed randomly between $0 \%$ and $10 \%$, (4) images were rotated randomly between $1^{\circ}$ and $90^{\circ}$, (5) images were randomly flipped or mirrored, and (6) images also were randomly sheared between $0 \%$ and $30 \%$. By performing augmentation through the above various methods, the features of each disease were trained at various positions and angles during model training. 
Table 2. The values of each argument used in ImageDataGenerator.

\begin{tabular}{ccc}
\hline & Argument & Value \\
\hline$(1)$ & Width_shift_range & 0.4 \\
\hline$(2)$ & Height_shift_range & 0.2 \\
\hline$(3)$ & Rotation_range & 90 \\
\hline$(4)$ & Zoom_range & 0.1 \\
\hline$(5)$ & $\begin{array}{c}\text { Horizontal_flip } \\
\text { Vertical_flip }\end{array}$ & True \\
\hline$(6)$ & Shear_range & 30 \\
\hline
\end{tabular}

\subsection{Preprocessing}

Original fundus images have two types of resolution, $2576 \times 1934$ pixel and $4496 \times 3000$ pixels with a 24-bit RGB channel. Each image was cropped to most of the circular part of the fundus and cropped to remove the black border. Then, each cropped image was resized to $512 \times 512$ pixels as input images for the AI models. Keras ImageDataGenerator (https: / / keras.io/ (accessed on 29 October 2020)) was used for the augmentation of preprocessed images during training.

\subsection{Convolutional Neural Network (CNN) Modeling}

Three CNN models for training were evaluated (Figure 1), Visual Geometry Group with 19 layers (VGG19) [21], GoogLeNet Inception v3 [22], and Deep Residual Learning for Image Recognition with 50 layers (ResNet50) [23], because these CNN models have been widely applied to various medical image classification tasks. These models are all similar in that they consist of convolutional and pooling layers arranged in sequential units and a final, fully connected layer (Figure 1A). A notable difference among the models is the use of nodes to perform additional operations. The Inception v3 model uses an inception node that reduces the amount of computation while using various convolutional filters (Figure 1B). The ResNet50 model uses a residual node that maps only information that needs to be additionally trained in the layer by connecting information trained in the previous layer to the current layer during training (Figure 1C). We loaded these models trained with image datasets from ImageNet (Accessed 29 October 2020. http:/ / www. image-net.org/) and trained the convolutional layers and fully connected layers with our fundus images. The dataset of macular image was randomly classified into five folds so that 5 -fold cross-validation could be performed to estimate model performance. Training was performed using multiple iterations with batch size of 4, learning rate of 0.00001 , and Adam optimization. This process was repeated for each model (VGG19, Inception v3, ResNet50) and cross-validation fold.

\subsection{Cross-Validation of AI-Based Diagnosis}

Cross-validation is a useful technique in evaluating the performance of deep learning models. In cross-validation, the dataset was randomly divided into training and test sets, with the training set used to build a model and the test set used to assess the performance of the model by measuring accuracy.

In k-fold cross-validation, the dataset was randomly divided into $\mathrm{k}$ subsets of equal size, with one used as a test set and the others as training sets. Cross-validation was performed $\mathrm{k}$ times to allow the use of all subsets exactly once as a test set. Model performance was determined according to the average model evaluation scores calculated across the $\mathrm{k}$ test subsets. Here, we evaluated the performance of the proposed CNN model using 5 -fold cross-validation, with performance determined according to the average accuracy of five cross-validations for each class comparison [2]. 
A

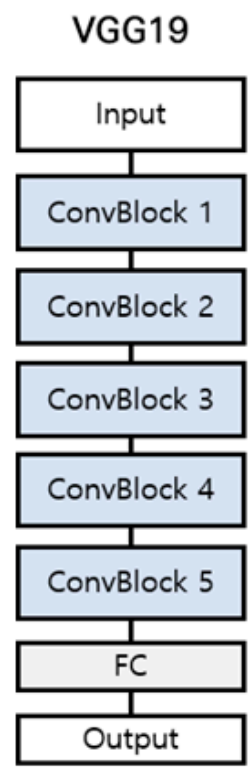

Inception V3
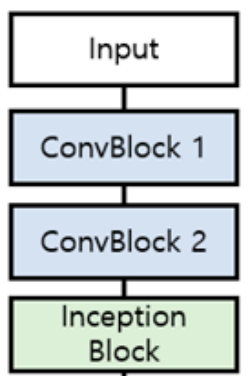

Block

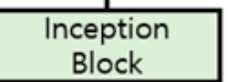

Block

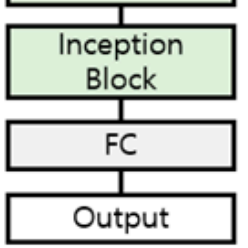

\section{ResNet50}

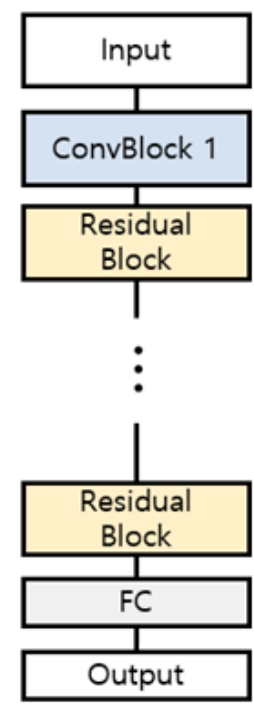

B

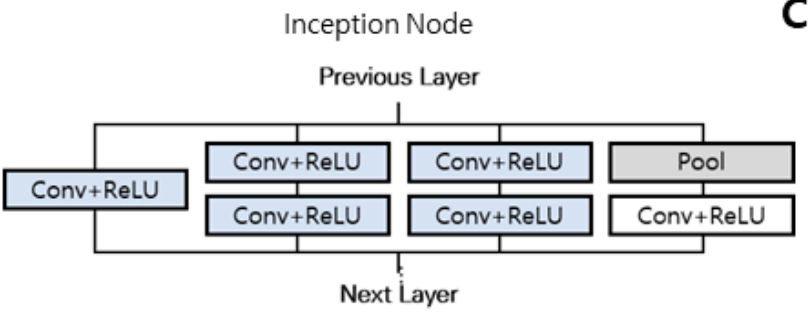

C Residual Node

Previous Layer

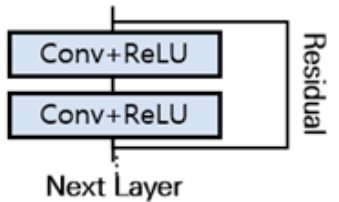

Figure 1. Comparison of three different convolutional neural network (CNN) architectures. (A) VGG19 model, Inception V3 model, and ResNet50 model. (B) Inception node uses various convolutional filters. (C) The residual node maps the necessary information using residual connection.

\subsection{Classification Performance Evaluation Index}

For diagnosis and accuracy calculation, it was necessary to categorize the model outputs, which are continuous scores. In one-class (normal vs. disease), since the output has a score between 0 and 1 , if it is less than 0.5 , it was diagnosed as 0 (normal), and if it is greater than or equal to 0.5 , it was diagnosed as 1 (disease). In nine-class diagnosis, the class with the highest probability was selected.

Accuracy, sensitivity, specificity, positive predictive value (PPV), and negative predictive value (NPV) were obtained as evaluation indices. Accuracy was defined as the proportion of correctly classified observations. Sensitivity was defined as the ratio of the total number of correctly classified positive examples divided to the total number of positive examples, whereas specificity was defined as the ratio of the total number of correctly classified negative examples divided to the total number of negative examples. PPV was defined as the proportion of predicted positives, which were actual positives, whereas NPV was defined as the proportion of predicted negative, which were actual negatives. The evaluation index was calculated for each class, corresponding to one class and all other classes. The formulas of each evaluation index were expressed as:

$$
\begin{gathered}
\text { Accuracy }=\frac{\mathrm{TP}+\mathrm{TN}}{\mathrm{TP}+\mathrm{TN}+\mathrm{FP}+\mathrm{FN}} \\
\text { Sensitivity }=\frac{\mathrm{TP}}{\mathrm{TP}+\mathrm{FN}}
\end{gathered}
$$




$$
\begin{gathered}
\text { Specificity }=\frac{\mathrm{TN}}{\mathrm{TN}+\mathrm{FP}} \\
\mathrm{PPV} \frac{\mathrm{TP}}{\mathrm{TP}+\mathrm{FP}} \\
\mathrm{NPV}=\frac{\mathrm{FP}}{\mathrm{TP}+\mathrm{FP}}
\end{gathered}
$$

where TP (true positive) is the number of samples correctly classified as positive, FN (false negative) is the number of samples incorrectly classified as negative, FP (false positive) is the number of samples incorrectly classified as positive, and TN (true negative) is the number of samples correctly classified as negative.

\section{Results}

\subsection{Two-Class Diagnosis}

We achieved a high accuracy for the two-class diagnosis between the normal condition and retinal diseases using 5-fold cross-validation. VGG19 CNN models showed the highest accuracy of $99.12 \%$ for two-class diagnosis (normal vs. disease, Table 3). These results suggest that our $\mathrm{CNN}$ models are suitable for the preliminary diagnosis process in ophthalmology.

Table 3. Comparison of outcomes of convolutional neural network models in two-class (normal vs. disease) diagnosis (accuracy).

\begin{tabular}{cccc}
\hline Model & VGG19 & Inception v3 & ResNet50 \\
\hline Accuracy & $99.12 \%$ & $98.08 \%$ & $97.85 \%$ \\
\hline
\end{tabular}

VGG19, Visual Geometry Group with 19 layers; ResNet50, Deep Residual Learning for Image Recognition with 50 layers.

\subsection{Nine-Class Diagnosis and Visualization}

Similar to two-class diagnosis, we performed nine-class diagnosis among the normal condition and eight other diseases using 5-fold cross-validation. Table 4 shows the combination performance of each $\mathrm{CNN}$ model and dense layer using 5-fold cross-validation. Each value shows the average test accuracy of all folds plus/minus standard deviation. Unlike two-class diagnostic results, ResNet50 CNN model with 128 nodes showed the highest accuracy of $87.42 \%$ for nine-class diagnosis.

Table 4. Accuracy results obtained using 5-fold cross-validation.

\begin{tabular}{cccc}
\hline Dense Layer & VGG19 & Inception v3 & ResNet50 \\
\hline 128 nodes & $0.8200 \pm 0.0282$ & $0.8340 \pm 0.0364$ & $0.8742 \pm 0.0349$ \\
256 nodes & $0.8135 \pm 0.0315$ & $0.8212 \pm 0.0444$ & $0.8646 \pm 0.0205$ \\
128 nodes +128 nodes & $0.8168 \pm 0.0243$ & $0.8360 \pm 0.0115$ & $0.8694 \pm 0.0338$ \\
256 nodes + 256 nodes & $0.8026 \pm 0.0365$ & $0.8483 \pm 0.0381$ & $0.8452 \pm 0.0351$ \\
\hline
\end{tabular}

The data are shown as mean \pm standard deviation. VGG19, Visual Geometry Group with 19 layers; ResNet50, Deep Residual Learning for Image Recognition with 50 layers.

For nine-class diagnosis, we finally selected and employed ResNet50 with 128 nodes (Table 5). Our AI tool showed $87.42 \%$ accuracy for the diagnosis among normal condition and various retinal diseases (nAMD, dAMD, ERM, RRD, RP, MH, RVO, and DR).

Gradient-weighted Class Activation Map (Grad-CAM) visualization was performed to identify areas showing the greatest effect of retinal diseases. Grad-CAM extracts feature maps of the final convolutional layer of each model trained using fundus images and computes the weights of the feature maps to represent the heatmap in the image (Figure 2). 
Table 5. Cross-validation results of classification performance evaluation index for nine-class diagnosis (accuracy, sensitivity, specificity, PPV, NPV).

\begin{tabular}{ccccccc}
\hline Model & Accuracy & Class & Sensitivity & Specificity & PPV & NPV \\
\hline & & dAMD & 0.8190 & 0.9844 & 0.8439 & 0.9770 \\
\cline { 2 - 6 } & & DR & 0.9262 & 0.9833 & 0.9052 & 0.9868 \\
\cline { 2 - 6 } & ERM & 0.9252 & 0.9830 & 0.9089 & 0.9850 \\
\cline { 2 - 6 } $\begin{array}{c}\text { ResNet50 } \\
\text { with 128 } \\
\text { nodes }\end{array}$ & $87.42 \%$ & MH & 0.8192 & 0.7960 & 0.7556 & 0.9861 \\
\cline { 2 - 6 } & & Normal & 0.8830 & 0.9873 & 0.9092 & 0.9800 \\
\cline { 2 - 6 } & RPD & 0.9085 & 0.9966 & 0.9600 & 0.9914 \\
\cline { 2 - 6 } & & 0.8143 & 0.9870 & 0.9125 & 0.9671 \\
\cline { 2 - 6 } & RVO & 0.8514 & 0.9916 & 0.8750 & 0.9882 \\
\hline
\end{tabular}

NPV, negative predictive value; PPV, positive predictive value; ResNet50, Deep Residual Learning for Image Recognition with 50 layers.

\section{A}

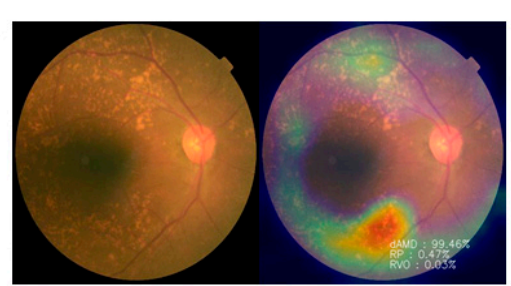

D

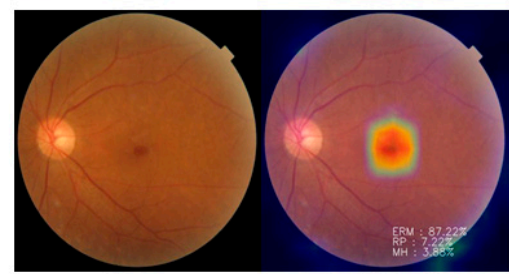

$\mathbf{G}$

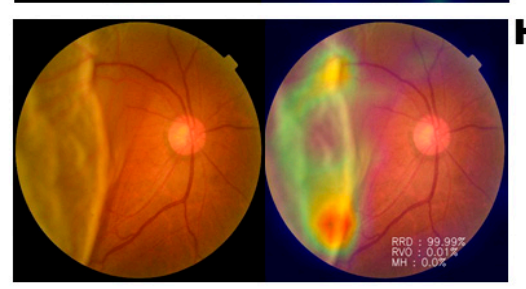

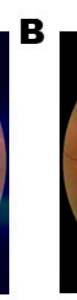

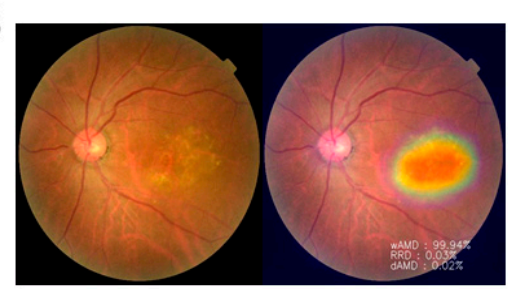

E
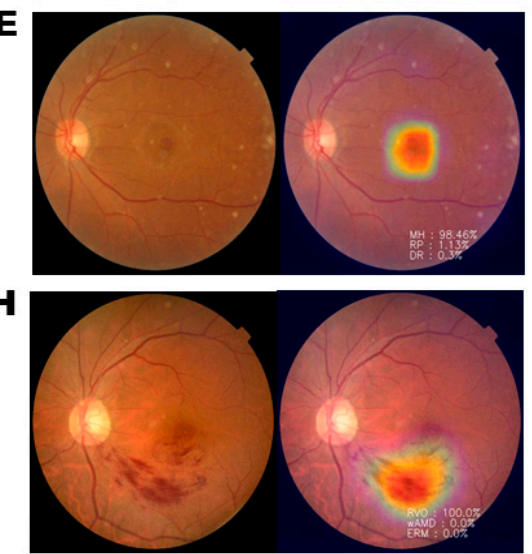

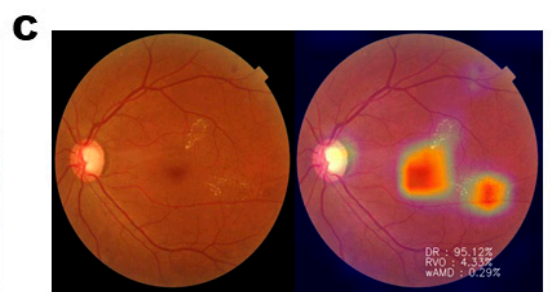

$\mathbf{F}$
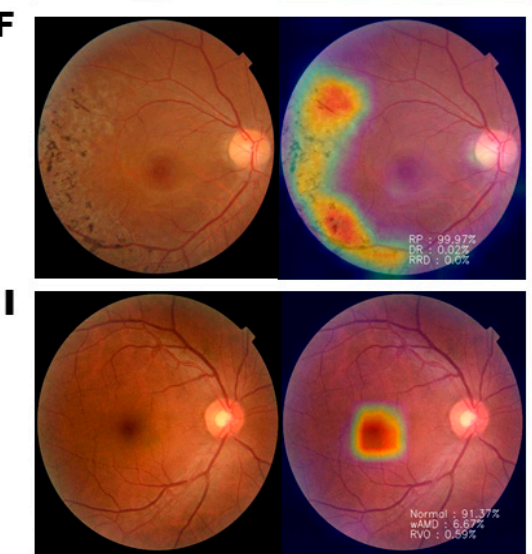

Figure 2. Examples of Gradient-weighted Class Activation Mapping (Grad-CAM) visualization of retinal diseases. GradCAM visualization of (A) dAMD, (B) wAMD, (C) DR, (D) ERM, (E) MH, (F) RP, (G) RRD, (H) RVO, and (I) normal retina. Grad-CAM extracts the feature map of the last convolution layer and shows a heatmap within the image describing the calculated weight of the feature map. Heatmap images of nAMD show that the AI tool identified pathological changes, such as drusen, bleeding, elevation of the center, pigmentation, surface wrinkling, and retinal detachment. However, in normal controls, the center of macula is identified, with no degenerated area.

\subsection{Classification Probability}

In the case of correct classification, when the fundus image with RP characteristics was classified using the trained model, it was classified as RP with $100 \%$ probability, and it was shown that symptoms were appropriately identified through the heatmap (Figure 3A). In the case of misclassification, nAMD was diagnosed as DR with 57.67\%, nAMD with $33.20 \%$, and RVO with $9.05 \%$, indicating that it was incorrectly diagnosed as DR (Figure 3B). However, it is possible to augment the diagnosis of ophthalmologists by referring to the identification area and proposed disease probability through the heatmap provided by our AI tool. 


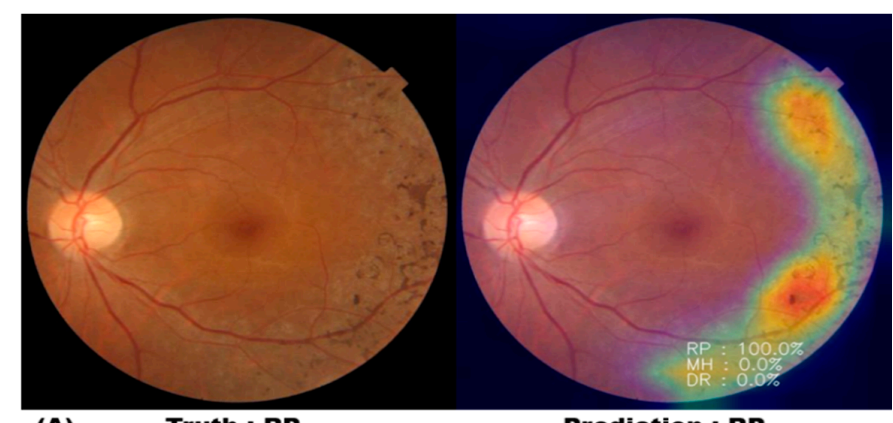

(A)

Truth : RP

Prediction : RP

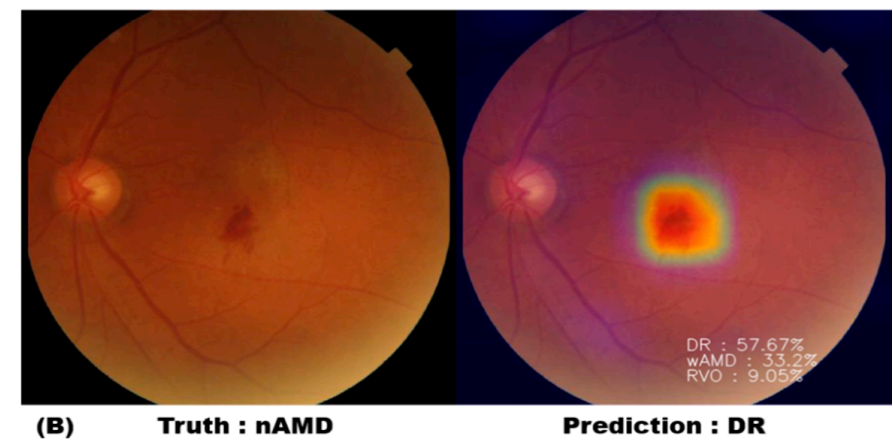

Figure 3. Heatmap and probability with correct classification and misclassification. (A) AI correctly diagnosed the RP fundus photograph as RP with 100\% probability. (B) AI diagnosed the nAMD fundus photograph with $57.67 \%$ of DR and $33.2 \%$ of nAMD.

\subsection{Comparison of Accuracy Values of the Deep Learning Diagnostic Tool and Residents in Ophthalmology}

To compare the performance between AI diagnosis and clinical reviewers, four ophthalmology residents of Ulsan University Hospital evaluated the fundus photographs and compared the results of evaluating fundus photographs with reference to AI results. A total of 180 fundus photographs were evaluated by extracting 20 images for each retinal disease. The 180 fundus photographs composed of eight types of retinal diseases and normal control group were randomly mixed, and each fundus photograph was read through a monitor for a sufficient time. At the second reading, the reading was performed while showing the result of the AI reading on the fundus photograph. A new AI model was trained by using the rest of the fundus photos as a training dataset, except for 180 photos, and we compared the results of the AI model's diagnosis of 180 fundus photos with the results of residents. The accuracy of AI was $83.9 \%$, which was lower than that of four ophthalmology residents. The accuracy of the results of diagnosis by four ophthalmology residents referring to the AI result slightly improved. However, residents showed reduced time spent by approximately $34 \%$ to $70 \%$ overall when they were notified of AI diagnosis results in advance (Table 6 ).

Table 6. Comparison of diagnostic results before and after AI reference. A total of 180 fundus photos were evaluated by extracting 20 images for each retinal disease.

\begin{tabular}{lccccccccc}
\hline & & \multicolumn{4}{c}{ Ophthalmology Residents' Results } \\
\cline { 3 - 10 } & AI Results & \multicolumn{3}{c}{ Before Referring to AI Results } & \multicolumn{3}{c}{ After Referring to AI Results } \\
\cline { 3 - 10 } & & $\mathbf{1}$ & $\mathbf{2}$ & $\mathbf{3}$ & $\mathbf{4}$ & $\mathbf{1}$ & $\mathbf{2}$ & $\mathbf{3}$ & $\mathbf{4}$ \\
\hline Wrong count & 29 & 15 & 21 & 28 & 27 & 14 & 17 & 29 & 23 \\
Accuracy (\%) & 83.9 & 91.7 & 88.3 & 84.4 & 85 & 92.2 & 90.6 & 83.9 & 87.2 \\
Time (min) & & 50 & 70 & 75 & 32 & 15 & 25 & 24 & 25 \\
\hline
\end{tabular}




\section{Discussion}

Deep learning AI-based diagnostic tools are currently adopted by medical experts. In ophthalmology, AI algorithms extracting fundus images of retinal pathologies are potentially suitable for the development of image-based diagnostic systems. The early differential diagnosis of retinal diseases, such as dAMD, nAMD, DR, ERM, RRD, RP, MH, and RVO, is critical for appropriate treatment [21]. In this study, we conducted two-class diagnosis with AI tool between normal condition and retinal diseases (eight diseases) and showed a high accuracy ( $>99 \%$ ) of VGG models. These results suggest that our AIbased tool is a promising approach for the preliminary diagnostic process. Furthermore, we developed an image-based AI diagnostic tool to detect and differentiate eight retinal diseases and normal control using fundus photograph.

Five-fold cross-validation revealed that the ResNet50 model had superior accuracy for nine-class classification compared with other models (VGG19 and Inception). Furthermore, the ResNet50 model with 128 nodes showed the highest accuracy $(>0.87)$. With this ResNet50 model, we compared the diagnostic performance of ophthalmology residents in the classification of nine retinal statuses (normal condition plus eight retinal diseases).

Four residents conducted differential diagnosis for nine classes and showed accuracy of $84.4 \%-91.7 \%$ without availability of preliminary outcome of the AI tool. However, they showed accuracy of $83.9 \%-92.2 \%$ when they could access the diagnostic outcome of the AI tool. Meanwhile, they shortened the time spent for diagnosis using AI tool's reference data of up to $51 \mathrm{~min}$ (from $75 \mathrm{~min}$ to $24 \mathrm{~min}$ ). Based on these results, the AI diagnostic model may be applicable to the preliminary process for an ophthalmologist's differential diagnosis.

Some retinal diseases are difficult to differentially diagnose based on fundus photograph only. For example, in the case of DR and RVO, it may be difficult to distinguish through fundus photograph only if it is not typical. Even in the case of $\mathrm{MH}$, accurate diagnosis may be difficult with fundus photograph only. In such diseases, additional patient information (medical history, medication, VA) and various types of retinal imaging examinations (OCT, FA/ICGA) are required for accurate diagnosis. However, it is difficult to demonstrate with an algorithm, and additional ophthalmic equipment is required. Therefore, to compensate for this shortcoming, we were able to increase the effectiveness of fundus diagnosis by showing the three high-priority diagnoses in order of $\%$ in the case of retinal diseases where differential diagnosis is difficult with fundus photograph only.

Some retinal diseases such as AMD and RP, which were also covered in this study, can be expressed in various forms by genetic changes related to RPE cells and photoreceptor cells $[22,23]$. From a future perspective, by expanding the area of application of the imagebased AI tools to these diseases, it is also useful for differential diagnosis of retinal diseases in phenotype-genotype association studies, which take into account mutations in already known causative genes and biochemical pathways related.

Our study has several limitations. First, the age distributions among subject groups did not match because the age at which the disease occurs differs according to the disease and a sufficient number of suitable fundus photographs were not secured. Second, the fundus photos used in our study have two types of resolution, and to minimize the difference, it was converted to the same resolution of $512 \times 512$ pixels through a preprocessing step and then used for AI learning. Although the resolution was identically matched through the preprocessing, there is a possibility that this process did not completely eliminate the difference between the two types of pictures. Third, with the help of our AI tool, the reading time was shortened, but the improvement in accuracy was not as high as the reduction in time. We also think the most important thing is to improve the accuracy of the diagnosis. However, we have shown that this study can help in the differential diagnosis of various retinal diseases through AI deep learning. 


\section{Conclusions}

Developing diagnostic tools for accurate diagnosis and prognostic prediction is required for the improvement of diagnostic accuracy and reduction of medical cost. Furthermore, AI-based platform and technology may resolve the low reliability of diagnostic imaging equipment in low-income countries. Therefore, we developed an image-based AI tool for differential diagnosis of retinal diseases using fundus photograph. These results suggest that our AI tool supports medical specialists to accurately diagnose retinal diseases in a cost-effective way.

Author Contributions: J.Y. and J.K.M. were responsible for study conception and design; T.-Y.H. and K.M.K. performed the experiments and data analyses; K.J.H., J.K., and A.K. participated in the discussion of the findings. All authors have read and agreed to the published version of the manuscript.

Funding: This work was supported by the National Research Foundation of Korea (NRF) grant, funded by the Korea government (MSIT) (No. 2017R1C1B5017929). This research was also supported by "Regional Innovation Strategy (RIS)" and Basic Science Research Program through the National Research Foundation of Korea (NRF) funded by the Ministry of Education (2017R1D1A3B03028084, 2019R1I1A3A01057696).

Institutional Review Board Statement: This study was reviewed, and the protocol was approved by the Institutional Human Experimentation Committee Review Board of Ulsan University Hospital, Ulsan, Republic of Korea (UUH 2020-07-036). The study was conducted in accordance with the ethical standards set forth in the 1964 Declaration of Helsinki.

Informed Consent Statement: This study was approved by the IRB of Ulsan University Hospital to waive the requirement to obtain any informed consent. Involves review of medical records of all patients who have undergone vitreoretinal surgery and/or visited the ophthalmology outpatient clinic for five years from 1 January 2015 to 30 June 2020, collecting limited data that will be doublecoded, so the link is known only to researchers. Results of the research will not affect the clinical care of the individuals, since this study is for diagnosis using already diagnosed data.

Data Availability Statement: The data generated during and/or analyzed the current study are available from the corresponding author on reasonable request.

Conflicts of Interest: The authors declare no conflict of interest.

\section{References}

1. Abramoff, M.D.; Lavin, P.T.; Birch, M.; Shah, N.; Folk, J.C. Pivotal trial of an autonomous AI-based diagnostic system for detection of diabetic retinopathy in primary care offices. NPJ Digit. Med. 2018, 1, 39. [CrossRef] [PubMed]

2. Heo, T.Y.; Kim, K.M.; Min, H.K.; Gu, S.M.; Kim, J.H.; Yun, J.; Min, J.K. Development of a deep-learning-based artificial intelligence tool for differential diagnosis between dry and neovascular age-related macular degeneration. Diagnostics 2020, 10, 261. [CrossRef]

3. Bernardes, R.; Serranho, P.; Lobo, C. Digital ocular fundus imaging: A review. Ophthalmologica 2011, 226, 161-181. [CrossRef]

4. Adhi, M.; Brewer, E.; Waheed, N.K.; Duker, J.S. Analysis of morphological features and vascular layers of choroid in diabetic retinopathy using spectral-domain optical coherence tomography. JAMA Ophthalmol. 2013, 131, 1267-1274. [CrossRef]

5. Hwang, T.S.; Gao, S.S.; Liu, L.; Lauer, A.K.; Bailey, S.T.; Flaxel, C.J.; Wilson, D.J.; Huang, D.; Jia, Y. Automated quantification of capillary nonperfusion using optical coherence tomography angiography in diabetic retinopathy. JAMA Ophthalmol. 2016, 134, 367-373. [CrossRef]

6. Hall, A. Recognising and managing diabetic retinopathy. Community Eye Health 2011, 24, 5-9.

7. Tremolada, G.; Del Turco, C.; Lattanzio, R.; Maestroni, S.; Maestroni, A.; Bandello, F. The role of angiogenesis in the development of proliferative diabetic retinopathy: Impact of intravitreal anti-VEGF treatment. Exp. Diabetes Res. 2012, 2012, 728325. [CrossRef]

8. McDonald, H.R.; Verre, W.P.; Aaberg, T.M. Surgical management of idiopathic epiretinal membranes. Ophthalmology 1986, 93, 978-983. [CrossRef]

9. Hillenkamp, J.; Saikia, P.; Gora, F.; Sachs, H.G.; Lohmann, C.P.; Roider, J.; Bäumler, W.; Gabel, V.-P. Macular function and morphology after peeling of idiopathic epiretinal membrane with and without the assistance of indocyanine green. Br. $J$. Ophthalmol. 2005, 89, 437-443. [CrossRef]

10. Falkner-Radler, C.I.; Glittenberg, C.; Hagen, S.; Benesch, T.; Binder, S. Spectral-domain optical coherence tomography for monitoring epiretinal membrane surgery. Ophthalmology 2010, 117, 798-805. [CrossRef]

11. Niwa, T.; Terasaki, H.; Kondo, M.; Piao, C.H.; Suzuki, T.; Miyake, Y. Function and morphology of macula before and after removal of idiopathic epiretinal membrane. Invest. Ophthalmol. Vis. Sci. 2003, 44, 1652-1656. [CrossRef] 
12. Schadlu, R.; Tehrani, S.; Shah, G.K.; Prasad, A.G. Long-term follow-up results of ilm peeling during vitrectomy surgery for premacular fibrosis. Retina 2008, 28, 853-857. [CrossRef]

13. Kuhn, F.; Aylward, B. Rhegmatogenous retinal detachment: A reappraisal of its pathophysiology and treatment. Ophthalmic Res. 2014, 51, 15-31. [CrossRef]

14. Levine, A.B.; Schlosser, C.; Grewal, J.; Coope, R.; Jones, S.J.M.; Yip, S. Rise of the machines: Advances in deep learning for cancer diagnosis. Trends Cancer 2019, 5, 157-169. [CrossRef]

15. Coudray, N.; Ocampo, P.S.; Sakellaropoulos, T.; Narula, N.; Snuderl, M.; Fenyo, D.; Moreira, A.L.; Razavian, N.; Tsirigos, A. Classification and mutation prediction from non-small cell lung cancer histopathology images using deep learning. Nat. Med. 2018, 24, 1559-1567. [CrossRef]

16. Gass, J.D. Idiopathic senile macular hole. Its early stages and pathogenesis. Arch. Ophthalmol. 1988, 106, 629-639. [CrossRef]

17. Chung, H.; Byeon, S.H. New insights into the pathoanatomy of macular holes based on features of optical coherence tomography. Surv. Ophthalmol. 2017, 62, 506-521. [CrossRef]

18. Scott, I.U.; VanVeldhuisen, P.C.; Ip, M.S.; Blodi, B.A.; Oden, N.L.; Awh, C.C. Effect of bevacizumab vs aflibercept on visual acuity among patients with macular edema due to central retinal vein occlusion: The SCORE2 randomized clinical trial. JAMA 2017, 317, 2072-2087. [CrossRef] [PubMed]

19. Ip, M.; Hendrick, A. Retinal vein occlusion review. Asia Pac. J. Ophthalmol 2018, 7, 40-45. [CrossRef]

20. Age-Related Eye Disease Study Research, G. A randomized, placebo-controlled, clinical trial of high-dose supplementation with vitamins $C$ and $E$ and beta carotene for age-related cataract and vision loss: AREDS report no. 9. Arch. Ophthalmol. 2001, 119, 1439-1452.

21. Muller, P.L.; Wolf, S.; Dolz-Marco, R.; Tafreshi, A.; Schmitz-Valckenberg, S.; Holz, F.G. Ophthalmic diagnostic imaging: Retina. In High. Resolution Imaging in Microscopy and Ophthalmology: New Frontiers in Biomedical Optics; Bille, J.F., Ed.; Springer: Cham, Switzerland, 2019; pp. 87-106.

22. Donato, L.; Scimone, C.; Alibrandi, S.; Pitruzzella, A.; Scalia, F.; D'Angelo, R.; Sidoti, A. Possible A2E Mutagenic Effects on RPE Mitochondrial DNA from Innovative RNA-Seq Bioinformatics Pipeline. Antioxidants 2020, 9, 1158. [CrossRef]

23. Scimone, C.; Alibrandi, S.; Scalinci, S.Z.; Battaglioda, E.T.; D’Angelo, R.; Sidoti, A.; Donato, L. Expression of Pro-Angiogenic Markers Is Enhanced by Blue Light in Human RPE Cells. Antioxidants 2020, 9, 1154. [CrossRef] 